\title{
The influence of substrate type and chlormequat on the growth and flowering of marigold (Tagetes L.)
}

\author{
Matgorzata Maślanka*, Renata Magdziarz \\ Department of Ornamental Plants \\ University of Agriculture in Krakow \\ 29 Listopada 54, 31-425 Kraków, Poland
}

\begin{abstract}
This study was conducted to investigate the effect of various horticultural substrates (compost, peat-coconut, peat TS1, flower soil, lowmoor peat) and a foliar spray of chlormequat (at a concentration of $1380 \mathrm{mg} \mathrm{dm}^{-3}$ ) on the growth and flowering of the marigold cultivars belonging to two species: Tagetes erecta - 'Marvel Mixture' and 'Taishan Orange', and Tagetes patula - 'Durango Red' and 'Bonanza Flame'. The obtained results show that the plants grown in peat TS1 and peat-coconut were taller, had longer internodes and leaves, and thicker stems than the plants grown in the other substrates. Chlormequat significantly reduced the height of 'Marvel Mixture' (in peat TS1), 'Taishan Orange' (in lowmoor peat) and 'Bonanza Flame' (in peat-coconut). The use of chlormequat also accelerated the development of flower heads in 'Taishan Orange' (in lowmoor peat).
\end{abstract}

Key words: annual plant, compost, flower soil, growth retardant, peat, peat-coconut

\section{INTRODUCTION}

Marigolds are among the most popular ornamental garden and balcony plants (Hongpakdee and Ruamrungsri 2015, Kumar et al. 2016). They are characterized by a very long flowering season, lasting from May until the autumn frosts, and high resistance to heat and drought. They occur naturally in subtropical South and Central America, and Mexico. The main species grown as garden plants are the Mexican marigold (Tagetes erecta), French marigold (T. patula) and Golden marigold (T. tenuifolia).

In mass production, marigold seedlings are usually grown for a specified date. Increased competition has led the industry to prepare seedlings of the highest possible quality, as only good quality plant material finds buyers nowadays.
The main factor affecting cultivation in containers is the properly prepared and selected substrate. The substrate determines both the correct root growth and the development of the aboveground parts. Each substrate should have appropriate physical characteristics, in particular high porosity, high water capacity, durable structure and availability of nutrients (Fascella 2015). Horticultural production is increasingly based on ready-made substrates produced on an industrial scale from single components that are strictly controlled in terms of quality. There are many readymade substrates, among which the peat-coconut substrate is particularly highly recommended by its manufacturers for the cultivation of balcony and bedding plants. However, the ready-made substrates based on peat and coconut fibre can be substituted

\footnotetext{
*Corresponding author.

Tel.: +48 1266252 49; fax: +48 1266252 66;

e-mail: m.maslanka@ogr.ur.krakow.pl (M. Maślanka).
} 
by cheaper compost-based soils (Ingelmo et al. 1998, Dobrowolska and Janicka 2014).

An important role in the cultivation of marigolds is also played by growth retardants, which influence the structure of plants, inhibiting their growth and often increasing the number of flowers (Latimer 1991, Abdella 2014). Retardants that are appropriately selected for the species and used in a correct concentration and at the right time during cultivation allow growers to obtain plants with specific characteristics affecting their decorative value (Asgarian et al. 2013).

This study focuses on two species: Tagetes erecta (Mexican marigold) and Tagetes patula (French marigold), which have the greatest economic importance among the available marigolds. The aim of this study was to examine the impact of various horticultural substrates and the plant growth retardant chlormequat on the growth and flowering of the studied marigold species.

\section{MATERIAL AND METHODS}

The experiment was carried out on four marigold cultivars belonging to two species: Tagetes erecta L. - 'Marvel Mixture' and 'Taishan Orange', and Tagetes patula L. - 'Durango Red' and 'Bonanza Flame', in the period from April to June of 2013. Seed material (rubbed seed) came from the seed company Vitroflora (Poland).

Marigold seeds were sown in rows, with a spacing of $2.0 \times 0.5 \mathrm{~cm}$, into plastic boxes, and covered with a layer of $1.5 \mathrm{~cm}$ of peat-coconut substrate produced by Ceres. The previous study (not published) had revealed peat-coconut to be the most suitable substrate for the germination of marigolds. Boxes were placed in a poly-tunnel, maintaining a day air temperature of $19-20^{\circ} \mathrm{C}$ and

Table 1. Individual macro- and micronutrients in Osmocote Exact fertiliser

\begin{tabular}{llc}
\hline Osmocote Exact & $\begin{array}{c}\text { Nutrient content } \\
(\%)\end{array}$ & $\begin{array}{c}\text { Microelements fully soluble in water } \\
(\%)\end{array}$ \\
\hline & $\mathrm{N}-15$ & $\mathrm{~B}-0.03$ \\
$\mathrm{NPK}(15+9+12)$ & $\mathrm{N}-\mathrm{NO}_{3}-7$ & $\mathrm{Cu}-0.050$ \\
$+\mathrm{MgO}(2.5)$ & $\mathrm{N}^{-\mathrm{NH}_{4}-8}$ & $\mathrm{Fe}-0.450$ \\
+ microelements & $\mathrm{P}_{2} \mathrm{O}_{5}-9$ & $\mathrm{Mn}-0.065$ \\
& $\mathrm{~K}_{2} \mathrm{O}-12$ & $\mathrm{Mo}-0.020$ \\
& $\mathrm{MgO}-2.5$ & $\mathrm{Zn}-0.015$ \\
\hline
\end{tabular}

Table 2. Composition of substrates with the following commercial names

\begin{tabular}{|c|c|c|c|}
\hline Substrate & $\mathrm{pH}$ in $\mathrm{H}_{2} \mathrm{O}$ & Salinity & Nutrients \\
\hline Compost + perlite $(3: 1)$ & 5.93 & $0.44 \mathrm{~g} \mathrm{NaCl} \mathrm{dm}^{-3}$ & $\begin{array}{l}79{\mathrm{mg} \mathrm{N}-\mathrm{NO}_{3} \mathrm{dm}^{-3}} \\
99 \mathrm{mg} \mathrm{P} \mathrm{dm}^{-3} \\
96 \mathrm{mg} \mathrm{K} \mathrm{dm}^{-3} \\
40 \mathrm{mg} \mathrm{Mg} \mathrm{dm}^{-3} \\
1034 \mathrm{mg} \mathrm{Ca} \mathrm{dm}^{-3}\end{array}$ \\
\hline $\begin{array}{l}\text { Peat-coconut } \\
\text { Ceres }\end{array}$ & $5.2-6.5$ & $1.2 \mathrm{~g} \mathrm{NaCl} \mathrm{dm}^{-3}$ & $\begin{array}{l}100-200 \mathrm{mg} \mathrm{N} \mathrm{dm}^{-3} \\
120-220 \mathrm{mg} \mathrm{P}_{2} \mathrm{O}_{5} \mathrm{dm}^{-3} \\
140-240 \mathrm{mg} \mathrm{K}_{2} \mathrm{O} \mathrm{dm}^{-3} \\
60-100 \mathrm{mg} \mathrm{Mg} \mathrm{dm}^{-3}\end{array}$ \\
\hline $\begin{array}{l}\text { Peat TS1 } \\
\text { Klasmann Deilmann }\end{array}$ & $5.5-6.5$ & $1.0 \mathrm{~g} \mathrm{NaCl} \mathrm{dm}^{-3}$ & $\begin{array}{l}280 \mathrm{mg} \mathrm{N} \mathrm{dm}^{-3} \\
200 \mathrm{mg} \mathrm{P}_{2} \mathrm{O}_{5} \mathrm{dm}^{-3} \\
360 \mathrm{mg} \mathrm{K}_{2} \mathrm{O} \mathrm{dm}^{-3} \\
100 \mathrm{mg} \mathrm{Mg} \mathrm{dm}^{-3}\end{array}$ \\
\hline Aura flower potting soil & 5.5 & $1.1 \mathrm{~g} \mathrm{NaCl} \mathrm{dm}^{-3}$ & $\begin{array}{l}84{\mathrm{mg} \mathrm{N}-\mathrm{NO}_{3} \mathrm{dm}^{-3}}_{76 \mathrm{mg} \mathrm{P} \mathrm{dm}^{-3}} \\
210 \mathrm{mg} \mathrm{K} \mathrm{dm}^{-3} \\
1110 \mathrm{mg} \mathrm{Ca} \mathrm{dm}^{-3} \\
92 \mathrm{mg} \mathrm{Mg} \mathrm{dm}^{-3}\end{array}$ \\
\hline Lowmoor peat & 6.0 & $0.18 \mathrm{~g} \mathrm{NaCl} \mathrm{dm}^{-3}$ & $\begin{array}{l}29{\mathrm{mg} \mathrm{N}-\mathrm{NO}_{3} \mathrm{dm}^{-3}} \\
0.389 \mathrm{mg} \mathrm{P} \mathrm{dm}^{-3} \\
0.51 \mathrm{mg} \mathrm{K} \mathrm{dm}^{-3} \\
792 \mathrm{mg} \mathrm{Ca} \mathrm{dm}^{-3} \\
37.2 \mathrm{mg} \mathrm{Mg} \mathrm{dm}^{-3} \\
38 \mathrm{mg} \mathrm{Cl} \mathrm{dm}^{-3}\end{array}$ \\
\hline
\end{tabular}


a night temperature of $17-18^{\circ} \mathrm{C}$. The seedlings were transplanted when the plants had developed four true leaves and reached $5 \mathrm{~cm}$ in height. Immediately before transplanting the seedlings, the bottom of each pot was filled with a small amount of the substrate and a single dose of Osmocote Exact (Tab. 1) fertilizer with a 5-6 month nutrient release period. For Tagetes erecta, the fertilizer was used in the amount of $3.5 \mathrm{~g} \mathrm{dm}^{-3}$ of substrate, and for Tagetes patula at $3 \mathrm{~g} \mathrm{dm}^{-3}$ of substrate, according to the fertilizing recommendations for bedding plants.

All of the cultivars were transplanted into plastic pots with a diameter of $10 \mathrm{~cm}$ and a volume of $374 \mathrm{~cm}^{3}$. Five different horticultural substrates were used (Tab. 2). Compost-based and lowmoor peat substrates were supplemented with ammonium nitrate and potassium sulphate to the level of 200 $\mathrm{mg} \mathrm{N}$ and $220 \mathrm{mg} \mathrm{K}_{2} \mathrm{O} \mathrm{dm} \mathrm{dm}^{-3}$. Lowmoor peat was additionally limed using calcium carbonate in the amount of $6 \mathrm{~g} \mathrm{dm}^{-3}$ to achieve $\mathrm{pH}=6.0$ (based on neutralisation curve and tested again using a System ISFET pH 3000, PNT 300 acidometer). At the same time, the air temperature in the poly-tunnel was reduced to $17^{\circ} \mathrm{C}$. Four weeks after transplanting, half of the plants were sprayed with chlormequat $(0.1 \%$ Cycocel $460 \mathrm{SL})$ at a concentration of $1380 \mathrm{mg} \mathrm{dm}^{-3}$. Plants not treated with chlormequat were used as the control (CTR).

The experiment was divided into two stages. In the first stage (Stage I), covering the three weeks between transplanting the seedlings and spraying them with the growth retardant, the influence of the various substrates on plant height, internode length, main stem diameter at the base, average length of leaves (growing on the main stem) and the number of flower buds per plant was assessed.

In the second stage (Stage II), lasting four weeks between spraying with the growth retardant and withering of the first flower heads, the measurements additionally included the number and diameter of developed inflorescences (flower heads) and, in the case of the French marigold, the number of shoots per plant.

Biometric measurements of the plants (during Stages I and II) were carried out using a ruler and a Vernier calliper. The length of the lower internode of Tagetes patula was measured only in the first stage of the experiment, as in the second stage the plants began to tiller, which was not observed for Tagetes erecta. The number of leaves increased with the growth of plants, from 4-8 (Stage I) to 19-22 leaves on average (Stage II). The flower bud counts give the total number of buds per plant, opening and closed. The flower head counts give the number of fully developed flower heads per plant and their diameter.

The experiment was performed in five replications with 20 plants each. Statistical analysis of the results consisted of analysis of variance in a two- and three-factor design. The comparison of means was carried out using the Tukey test, with the significance level $p \leq 0.05$.

\section{RESULTS}

\section{Stage I - Effect of substrate type on the growth and development of marigolds}

The analysis of results at 21 days after transplanting the Mexican marigold demonstrated that the nature

Table 3. Effect of genotype and type of substrate on the growth and development of Tagetes erecta 21 days after transplanting

\begin{tabular}{|c|c|c|c|c|c|c|}
\hline \multirow[b]{2}{*}{ Cultivar } & \multirow[b]{2}{*}{ Substrate } & \multicolumn{5}{|c|}{ Growth parameter } \\
\hline & & $\begin{array}{l}\text { Plant height } \\
(\mathrm{cm})\end{array}$ & $\begin{array}{l}\text { Leaf length } \\
\quad(\mathrm{mm})\end{array}$ & $\begin{array}{c}\text { Internode length } \\
(\mathrm{mm})\end{array}$ & $\begin{array}{l}\text { Stem diameter } \\
(\mathrm{mm})\end{array}$ & $\begin{array}{l}\text { Number } \\
\text { of buds }\end{array}$ \\
\hline Taishan Orange & \multirow{2}{*}{ Compost } & $10.4 \mathrm{~cd}^{*}$ & $37.0 \mathrm{bc}$ & $9.2 \mathrm{~d}$ & $4.5 \mathrm{~cd}$ & $0.75 \mathrm{~b}$ \\
\hline Marvel Mixture & & $10.3 \mathrm{~cd}$ & $37.1 \mathrm{bc}$ & $8.1 \mathrm{e}$ & $4.8 \mathrm{bc}$ & $0.90 \mathrm{a}$ \\
\hline Taishan Orange & \multirow{2}{*}{ Peat-coconut } & $12.6 \mathrm{a}$ & $40.7 \mathrm{a}$ & $12.0 \mathrm{~b}$ & $6.1 \mathrm{a}$ & $0.00 \mathrm{f}$ \\
\hline Marvel Mixture & & $12.4 \mathrm{ab}$ & $41.0 \mathrm{a}$ & $12.9 \mathrm{a}$ & $5.7 \mathrm{ab}$ & $0.00 \mathrm{f}$ \\
\hline Taishan Orange & \multirow{2}{*}{ Peat TS1 } & $11.3 \mathrm{bc}$ & $40.6 \mathrm{a}$ & $10.1 \mathrm{~cd}$ & $6.6 \mathrm{a}$ & $0.30 \mathrm{de}$ \\
\hline Marvel Mixture & & $11.6 \mathrm{ab}$ & $41.6 \mathrm{a}$ & $10.2 \mathrm{c}$ & $6.3 \mathrm{a}$ & $0.00 \mathrm{f}$ \\
\hline Taishan Orange & \multirow{2}{*}{ Flower soil } & $9.5 \mathrm{~d}$ & $39.3 \mathrm{ab}$ & $9.9 \mathrm{~cd}$ & $4.7 \mathrm{c}$ & $0.60 \mathrm{c}$ \\
\hline Marvel Mixture & & $10.2 \mathrm{~cd}$ & $37.6 \mathrm{bc}$ & $10.1 \mathrm{c}$ & $5.1 \mathrm{bc}$ & $0.40 \mathrm{~d}$ \\
\hline Taishan Orange & \multirow{2}{*}{ Lowmoor peat } & $9.4 \mathrm{~d}$ & $33.6 \mathrm{~d}$ & $9.4 \mathrm{~cd}$ & $3.8 \mathrm{de}$ & $0.25 \mathrm{e}$ \\
\hline Marvel Mixture & & $10.0 \mathrm{~d}$ & $36.7 \mathrm{c}$ & $9.7 \mathrm{~cd}$ & $3.6 \mathrm{e}$ & $0.35 \mathrm{de}$ \\
\hline
\end{tabular}

*Means marked with the same letter do not differ significantly at the significance level $p \leq 0.05$ 
Table 4. Effect of genotype and type of substrate on the growth and development of Tagetes patula 21 days after transplanting

\begin{tabular}{lcccccc}
\hline \multirow{2}{*}{ Cultivar } & Substrate & \multicolumn{5}{c}{ Growth parameter } \\
\cline { 3 - 7 } & \multirow{2}{*}{ Compost } & $\begin{array}{c}\text { Plant height } \\
(\mathrm{cm})\end{array}$ & $\begin{array}{c}\text { Leaf length } \\
(\mathrm{mm})\end{array}$ & $\begin{array}{c}\text { Internode length } \\
(\mathrm{mm})\end{array}$ & $\begin{array}{c}\text { Stem diameter } \\
(\mathrm{mm})\end{array}$ & $\begin{array}{c}\text { Number } \\
\text { of buds }\end{array}$ \\
\hline Bonanza Flame & $9.5 \mathrm{bc}^{*}$ & $35.3 \mathrm{c}$ & $8.3 \mathrm{~d}-\mathrm{f}$ & $3.9 \mathrm{bc}$ & $1.00 \mathrm{a}$ \\
Durango Red & \multirow{2}{*}{ Peat-coconut } & $11.0 \mathrm{a}$ & $36.0 \mathrm{bc}$ & $8.6 \mathrm{~cd}$ & $3.6 \mathrm{~cd}$ & $0.90 \mathrm{ab}$ \\
\hline Bonanza Flame & $10.5 \mathrm{ab}$ & $37.6 \mathrm{a}-\mathrm{c}$ & $11.8 \mathrm{a}$ & $4.6 \mathrm{a}$ & $0.80 \mathrm{~b}$ \\
Durango Red & \multirow{2}{*}{ Peat TS1 } & $10.5 \mathrm{ab}$ & $38.2 \mathrm{ab}$ & $9.1 \mathrm{~b}-\mathrm{d}$ & $4.4 \mathrm{ab}$ & $0.90 \mathrm{ab}$ \\
\hline Bonanza Flame & $10.0 \mathrm{~b}$ & $38.7 \mathrm{a}$ & $9.2 \mathrm{bc}$ & $4.6 \mathrm{a}$ & $0.85 \mathrm{~b}$ \\
Durango Red & \multirow{2}{*}{ Flower soil } & $8.9 \mathrm{~cd}$ & $37.7 \mathrm{a}-\mathrm{c}$ & $8.4 \mathrm{c}-\mathrm{f}$ & $4.3 \mathrm{ab}$ & $1.00 \mathrm{a}$ \\
Bonanza Flame & $8.8 \mathrm{~cd}$ & $38.3 \mathrm{ab}$ & $7.7 \mathrm{ef}$ & $4.6 \mathrm{a}$ & $0.65 \mathrm{c}$ \\
Durango Red & \multirow{2}{*}{ Lowmoor peat } & $9.1 \mathrm{~cd}$ & $31.1 \mathrm{~d}$ & $8.2 \mathrm{ef}$ & $3.3 \mathrm{~d}$ & $0.20 \mathrm{e}$ \\
\hline Bonanza Flame & $8.4 \mathrm{~d}$ & $27.8 \mathrm{e}$ & $7.5 \mathrm{f}$ & $3.3 \mathrm{~d}$ & $0.50 \mathrm{~d}$ \\
Durango Red & & & & & \\
\hline
\end{tabular}

*Explanations: see Table 3

of the substrate affected the grow th and development of both cultivars tested. Taller plants were found on the peat TS1 and peat-coconut substrates (11.3-12.6 $\mathrm{cm})$ as compared to the plants grown in the other substrates $(9.4-10.4 \mathrm{~cm})$ (Tab. 3). A similar pattern was observed for leaf length and stem diameter, which in both cultivars were also higher for the peat TS1 and peat-coconut substrates. The longest internodes (12.0 and $12.9 \mathrm{~mm}$ ) were recorded for the plants grown in the peat-coconut substrate. Using the lowmoor peat substrate resulted in plants that grew more slowly, had shorter leaves and thinner stems in comparison with the plants grown in the other substrates (Tab. 3). Genotypic differences in the development of the tested plants were observable in the length of the leaves in lowmoor peat, and in the length of the internodes in the compost soil and the peat-coconut substrate.

At 21 days after transplanting, the setting of flower buds was observed for both marigold cultivars. The highest number of flower buds $(0.90)$ developed on the Marvel Mixture cultivar grown in the compost substrate. No buds formed on any plants grown in the peat-coconut substrate, while 'Marvel Mixture' did not develop any buds when grown in the peat TS1 substrate. For the other substrates, the number of flower buds was in the range of 0.25-0.60 (Tab. 3). All of the buds were in the closed bud developmental phase.

At three weeks after transplanting, a positive effect of peat TS1 and peat-coconut substrates on the growth of both cultivars of French marigold was observed, with an average height of approximately $10.0-11.0 \mathrm{~cm}$ (Tab. 4). Leaf length and stem diameter were greater, $37.7-38.3 \mathrm{~mm}$ and 4.3-4.6 $\mathrm{mm}$, respectively, for plants grown in the flower soil, in comparison with the other substrates. The longest internodes were obtained for the Durango Red cultivar grown in the peatcoconut substrate. These internodes were $2.1 \mathrm{~cm}$ longer than the internodes of the Bonanza Flame cultivar grown in the same medium. The shortest leaves $(27.8 \mathrm{~mm})$ were recorded for 'Durango Red' grown in lowmoor peat. Lower values for leaf length and shoot diameter were recorded for both cultivars grown in lowmoor peat, as compared to plants grown in the other substrates. For 'Durango Red', also lower height was observed in lowmoor peat than in peat TS1, peat-coconut and compost (Tab. 4).

After 21 days from transplanting, the formation and opening of flower buds was observed for the French marigold. More buds (1.00) were noted for 'Bonanza Flame' cultivated in compost-based soil, peat TS1 and flower soil. The same cultivar grown in lowmoor peat formed the lowest number of buds (0.20) (Tab. 4).

\section{Stage II - Effect of substrate type and growth retardant on the growth and development of marigolds}

Regardless of the cultivar, seven weeks after being transplanted into lowmoor peat, the Mexican marigold plants were characterized by the lowest height $(23.3-25.1 \mathrm{~cm})$ and the thinnest stems $(7.5$ 7.9) in comparison with the plants grown in the other substrates (Tab. 5). Average leaf length was at a similar low level (47.4-58.3), both in compost and lowmoor peat. Longer leaves and thicker stems were obtained in the peat TS1 substrate (although 
Table 5. Influence of genotype, type of substrate and chlormequat on the growth and development of Tagetes erecta four weeks after spraying with growth retardant (49 days after transplanting)

\begin{tabular}{|c|c|c|c|c|c|}
\hline Cultivar & Substrate & Chlormequat & $\begin{array}{l}\text { Plant height } \\
(\mathrm{cm})\end{array}$ & $\begin{array}{l}\text { Leaf length } \\
\text { (mm) }\end{array}$ & $\begin{array}{l}\text { Stem diameter } \\
(\mathrm{mm})\end{array}$ \\
\hline \multirow{2}{*}{ Taishan Orange } & \multirow{4}{*}{ Compost } & CTR*** & $28.0 \mathrm{ab}^{*}$ & $56.0 \mathrm{~d}-\mathrm{f}$ & $10.0 \mathrm{c}-\mathrm{e}$ \\
\hline & & $\mathrm{R}^{* *}$ & $25.6 \mathrm{~b}-\mathrm{f}$ & $47.4 \mathrm{f}$ & $10.1 \mathrm{c}-\mathrm{e}$ \\
\hline \multirow{2}{*}{ Marvel Mixture } & & CTR & $26.7 \mathrm{a}-\mathrm{e}$ & $57.1 \mathrm{~b}-\mathrm{f}$ & $10.9 \mathrm{a}-\mathrm{c}$ \\
\hline & & $\mathrm{R}$ & $24.1 \mathrm{e}-\mathrm{g}$ & $58.3 \mathrm{a}-\mathrm{f}$ & $10.3 \mathrm{c}-\mathrm{e}$ \\
\hline \multirow{2}{*}{ Taishan Orange } & \multirow{4}{*}{ Peat-coconut } & CTR & $28.1 \mathrm{ab}$ & 63.4 a-e & $10.5 \mathrm{~b}-\mathrm{e}$ \\
\hline & & $\mathrm{R}$ & $26.0 \mathrm{a}-\mathrm{f}$ & 61.9 a-e & $10.1 \mathrm{c}-\mathrm{e}$ \\
\hline \multirow{2}{*}{ Marvel Mixture } & & CTR & $27.2 \mathrm{a}-\mathrm{d}$ & 64.7 a-e & $10.3 \mathrm{c}-\mathrm{e}$ \\
\hline & & $\mathrm{R}$ & $25.4 \mathrm{~b}-\mathrm{f}$ & 65.3 a-e & $10.5 \mathrm{~b}-\mathrm{e}$ \\
\hline \multirow{2}{*}{ Taishan Orange } & \multirow{4}{*}{ Peat TS1 } & CTR & 27.9 a-c & 69.2 a-d & 10.6 b-d \\
\hline & & $\mathrm{R}$ & $25.2 \mathrm{c}-\mathrm{f}$ & $70.6 \mathrm{a}-\mathrm{c}$ & $10.3 \mathrm{c}-\mathrm{e}$ \\
\hline \multirow{2}{*}{ Marvel Mixture } & & CTR & $27.0 \mathrm{a}-\mathrm{d}$ & $71.1 \mathrm{ab}$ & $11.9 \mathrm{a}$ \\
\hline & & $\mathrm{R}$ & $24.1 \mathrm{e}-\mathrm{g}$ & $71.3 \mathrm{a}$ & $11.6 \mathrm{ab}$ \\
\hline \multirow{2}{*}{ Taishan Orange } & \multirow{4}{*}{ Flower soil } & CTR & $27.9 \mathrm{a}-\mathrm{c}$ & $63.1 \mathrm{a}-\mathrm{e}$ & $9.6 \mathrm{de}$ \\
\hline & & $\mathrm{R}$ & $25.8 \mathrm{~b}-\mathrm{f}$ & $64.0 \mathrm{a}-\mathrm{e}$ & $9.4 \mathrm{e}$ \\
\hline \multirow{2}{*}{ Marvel Mixture } & & CTR & $28.7 \mathrm{a}$ & $62.6 \mathrm{a}-\mathrm{e}$ & $9.6 \mathrm{de}$ \\
\hline & & $\mathrm{R}$ & $26.0 \mathrm{a}-\mathrm{f}$ & $63.3 \mathrm{a}-\mathrm{e}$ & $9.5 \mathrm{e}$ \\
\hline \multirow{2}{*}{ Taishan Orange } & \multirow{4}{*}{ Lowmoor peat } & CTR & $25.1 \mathrm{~d}-\mathrm{f}$ & $51.7 \mathrm{ef}$ & $7.5 \mathrm{f}$ \\
\hline & & $\mathrm{R}$ & $22.0 \mathrm{~g}$ & $47.4 \mathrm{f}$ & $7.3 \mathrm{f}$ \\
\hline \multirow{2}{*}{ Marvel Mixture } & & CTR & $23.3 \mathrm{fg}$ & $55.5 \mathrm{~d}-\mathrm{f}$ & $7.9 \mathrm{f}$ \\
\hline & & $\mathrm{R}$ & $22.0 \mathrm{~g}$ & $56.6 \mathrm{c}-\mathrm{f}$ & $7.6 \mathrm{f}$ \\
\hline
\end{tabular}

*Explanations: see Table $3, * * \mathrm{R}-$ chlormequat, $* * * \mathrm{CTR}-$ control

Table 6. Influence of genotype, type of substrate and growth retardant on the inflorescence of Tagetes erecta four weeks after spraying with growth retardant (49 days after transplanting)

\begin{tabular}{|c|c|c|c|c|c|}
\hline Cultivar & Substrate & Chlormequat & $\begin{array}{c}\text { Number of } \\
\text { buds }\end{array}$ & $\begin{array}{c}\text { Number of } \\
\text { flower heads }\end{array}$ & $\begin{array}{c}\text { Flower head } \\
\text { diameter }(\mathrm{cm})\end{array}$ \\
\hline \multirow{2}{*}{ Taishan Orange } & \multirow{4}{*}{ Compost } & $\mathrm{CTR}^{* * *}$ & $2.30 \mathrm{a}^{*}$ & $2.2 \mathrm{ab}$ & $7.10 \mathrm{a}-\mathrm{f}$ \\
\hline & & $\mathrm{R} * *$ & $1.70 \mathrm{a}-\mathrm{d}$ & $2.1 \mathrm{ab}$ & $6.60 \mathrm{~d}-\mathrm{h}$ \\
\hline \multirow{2}{*}{ Marvel Mixture } & & CTR & $1.40 \mathrm{a}-\mathrm{d}$ & $1.0 \mathrm{f}$ & $7.40 \mathrm{a}-\mathrm{d}$ \\
\hline & & $\mathrm{R}$ & $1.90 \mathrm{a}-\mathrm{c}$ & $1.0 \mathrm{f}$ & $6.90 \mathrm{c}-\mathrm{g}$ \\
\hline \multirow{2}{*}{ Taishan Orange } & \multirow{4}{*}{ Peat-coconut } & CTR & $2.10 \mathrm{ab}$ & $1.8 \mathrm{a}-\mathrm{d}$ & $6.50 \mathrm{e}-\mathrm{h}$ \\
\hline & & $\mathrm{R}$ & $2.00 \mathrm{ab}$ & $1.7 \mathrm{a}-\mathrm{e}$ & $6.30 \mathrm{f}-\mathrm{h}$ \\
\hline \multirow{2}{*}{ Marvel Mixture } & & CTR & $2.00 \mathrm{ab}$ & $1.8 \mathrm{a}-\mathrm{d}$ & $7.50 \mathrm{a}-\mathrm{d}$ \\
\hline & & $\mathrm{R}$ & $1.90 \mathrm{a}-\mathrm{c}$ & $1.9 \mathrm{a}-\mathrm{c}$ & $7.40 \mathrm{a}-\mathrm{d}$ \\
\hline \multirow{2}{*}{ Taishan Orange } & \multirow{4}{*}{ Peat TS1 } & CTR & $2.30 \mathrm{a}$ & $2.3 \mathrm{a}$ & $7.70 \mathrm{a}-\mathrm{c}$ \\
\hline & & $\mathrm{R}$ & $2.00 \mathrm{ab}$ & $2.0 \mathrm{a}-\mathrm{c}$ & $7.50 \mathrm{a}-\mathrm{d}$ \\
\hline \multirow{2}{*}{ Marvel Mixture } & & CTR & $1.50 \mathrm{a}-\mathrm{d}$ & $1.9 \mathrm{a}-\mathrm{c}$ & $8.20 \mathrm{a}$ \\
\hline & & $\mathrm{R}$ & $2.30 \mathrm{a}$ & $1.8 \mathrm{a}-\mathrm{d}$ & $8.00 \mathrm{ab}$ \\
\hline \multirow{2}{*}{ Taishan Orange } & \multirow{4}{*}{ Flower soil } & CTR & $1.60 \mathrm{a}-\mathrm{d}$ & $1.0 \mathrm{f}$ & $7.20 \mathrm{~b}-\mathrm{f}$ \\
\hline & & $\mathrm{R}$ & $1.50 \mathrm{a}-\mathrm{d}$ & $1.0 \mathrm{f}$ & $7.10 \mathrm{~b}-\mathrm{f}$ \\
\hline \multirow{2}{*}{ Marvel Mixture } & & CTR & $1.00 \mathrm{~cd}$ & $1.2 \mathrm{ef}$ & $7.10 \mathrm{~b}-\mathrm{f}$ \\
\hline & & $\mathrm{R}$ & $0.80 \mathrm{~d}$ & $1.3 \mathrm{~d}-\mathrm{f}$ & $7.00 \mathrm{c}-\mathrm{g}$ \\
\hline \multirow{2}{*}{ Taishan Orange } & \multirow{4}{*}{ Lowmoor peat } & CTR & $0.90 \mathrm{~cd}$ & $1.0 \mathrm{f}$ & $6.00 \mathrm{gh}$ \\
\hline & & $\mathrm{R}$ & $1.40 \mathrm{a}-\mathrm{d}$ & $1.8 \mathrm{a}-\mathrm{d}$ & $5.70 \mathrm{~h}$ \\
\hline \multirow{2}{*}{ Marvel Mixture } & & CTR & $1.40 \mathrm{a}-\mathrm{d}$ & $1.0 \mathrm{f}$ & $6.30 \mathrm{f}-\mathrm{h}$ \\
\hline & & $\mathrm{R}$ & $1.30 \mathrm{~b}-\mathrm{d}$ & $1.5 \mathrm{c}-\mathrm{f}$ & $6.20 \mathrm{f}-\mathrm{h}$ \\
\hline
\end{tabular}

$*, * *, * * *$ Explanations: see Tabs 3-5 
Table 7. Influence of genotype, type of substrate and growth retardant on the growth and development of Tagetes patula four weeks after spraying with growth retardant (49 days after transplanting)

\begin{tabular}{|c|c|c|c|c|c|c|}
\hline Cultivar & Substrate & Chlormequat & $\begin{array}{l}\text { Plant height } \\
(\mathrm{cm})\end{array}$ & $\begin{array}{l}\text { Leaf length } \\
(\mathrm{mm})\end{array}$ & $\begin{array}{l}\text { Stem diameter } \\
(\mathrm{mm})\end{array}$ & $\begin{array}{l}\text { Number } \\
\text { of shoots }\end{array}$ \\
\hline \multirow{2}{*}{ Bonanza Flame } & \multirow{4}{*}{ Compost } & $\mathrm{CTR}^{* * *}$ & 19.1 a-d* & $45.6 \mathrm{f}-\mathrm{h}$ & $7.6 \mathrm{~d}-\mathrm{f}$ & $2.61 \mathrm{ab}$ \\
\hline & & $\mathrm{R} * *$ & $17.7 \mathrm{~b}-\mathrm{d}$ & $46.6 \mathrm{e}-\mathrm{h}$ & $7.5 \mathrm{~d}-\mathrm{g}$ & $2.78 \mathrm{a}$ \\
\hline \multirow{2}{*}{ Durango Red } & & CTR & $20.6 \mathrm{a}-\mathrm{c}$ & $45.0 \mathrm{~h}$ & $7.8 \mathrm{c}-\mathrm{f}$ & $2.69 \mathrm{ab}$ \\
\hline & & $\mathrm{R}$ & $18.1 \mathrm{~b}-\mathrm{d}$ & $45.8 \mathrm{f}-\mathrm{h}$ & $7.5 \mathrm{~d}-\mathrm{g}$ & $2.80 \mathrm{a}$ \\
\hline \multirow{2}{*}{ Bonanza Flame } & \multirow{4}{*}{ Peat-coconut } & CTR & $22.3 \mathrm{a}$ & $50.0 \mathrm{~d}-\mathrm{f}$ & $9.3 \mathrm{a}$ & $2.58 \mathrm{a}-\mathrm{c}$ \\
\hline & & $\mathrm{R}$ & $16.1 \mathrm{~d}$ & $53.2 \mathrm{~d}-\mathrm{f}$ & $9.2 \mathrm{ab}$ & $3.00 \mathrm{a}$ \\
\hline \multirow{2}{*}{ Durango Red } & & CTR & $20.8 \mathrm{a}-\mathrm{c}$ & $52.8 \mathrm{~cd}$ & $8.7 \mathrm{a}-\mathrm{c}$ & $2.50 \mathrm{a}-\mathrm{c}$ \\
\hline & & $\mathrm{R}$ & $18.5 \mathrm{a}-\mathrm{d}$ & $57.7 \mathrm{ab}$ & $8.2 \mathrm{~b}-\mathrm{e}$ & $3.00 \mathrm{a}$ \\
\hline \multirow{2}{*}{ Bonanza Flame } & \multirow{4}{*}{ Peat TS1 } & CTR & $19.5 \mathrm{a}-\mathrm{d}$ & $57.8 \mathrm{ab}$ & $9.1 \mathrm{ab}$ & $2.78 \mathrm{a}$ \\
\hline & & $\mathrm{R}$ & $18.7 \mathrm{a}-\mathrm{d}$ & $58.6 \mathrm{a}$ & $8.7 \mathrm{a}-\mathrm{c}$ & $2.88 \mathrm{a}$ \\
\hline \multirow{2}{*}{ Durango Red } & & CTR & $19.0 \mathrm{a}-\mathrm{d}$ & $55.8 \mathrm{a}-\mathrm{c}$ & $9.2 \mathrm{ab}$ & $2.78 \mathrm{a}$ \\
\hline & & $\mathrm{R}$ & $18.2 \mathrm{~b}-\mathrm{d}$ & $56.4 \mathrm{a}-\mathrm{c}$ & $9.0 \mathrm{ab}$ & $3.00 \mathrm{a}$ \\
\hline \multirow{2}{*}{ Bonanza Flame } & \multirow{4}{*}{ Flower soil } & CTR & $19.4 \mathrm{a}-\mathrm{d}$ & $50.1 \mathrm{~d}-\mathrm{f}$ & $8.4 \mathrm{a}-\mathrm{e}$ & $2.72 \mathrm{ab}$ \\
\hline & & $\mathrm{R}$ & $18.1 \mathrm{~b}-\mathrm{d}$ & $49.8 \mathrm{~d}-\mathrm{g}$ & $8.5 \mathrm{a}-\mathrm{d}$ & $2.80 \mathrm{a}$ \\
\hline \multirow{2}{*}{ Durango Red } & & CTR & $19.2 \mathrm{a}-\mathrm{d}$ & $50.0 \mathrm{~d}-\mathrm{f}$ & $8.6 \mathrm{a}-\mathrm{d}$ & $2.91 \mathrm{a}$ \\
\hline & & $\mathrm{R}$ & $17.6 \mathrm{~b}-\mathrm{d}$ & $50.7 \mathrm{de}$ & $8.5 \mathrm{a}-\mathrm{d}$ & $3.00 \mathrm{a}$ \\
\hline \multirow{2}{*}{ Bonanza Flame } & \multirow{4}{*}{ Lowmoor peat } & CTR & $21.4 \mathrm{ab}$ & $44.0 \mathrm{~h}$ & $6.5 \mathrm{~g}$ & $2.11 \mathrm{bc}$ \\
\hline & & $\mathrm{R}$ & $18.5 \mathrm{a}-\mathrm{d}$ & $44.6 \mathrm{~h}$ & $6.8 \mathrm{fg}$ & $2.58 \mathrm{a}-\mathrm{c}$ \\
\hline \multirow{2}{*}{ Durango Red } & & CTR & 18.9 a-d & $44.3 \mathrm{~h}$ & $7.8 \mathrm{c}-\mathrm{f}$ & $1.97 \mathrm{c}$ \\
\hline & & $\mathrm{R}$ & $17.3 \mathrm{~cd}$ & $45.1 \mathrm{gh}$ & $7.4 \mathrm{e}-\mathrm{g}$ & $2.61 \mathrm{ab}$ \\
\hline
\end{tabular}

$*, * *, * * *$ Explanations: see Tabs 3-5

Table 8. Influence of genotype, type of substrate and growth retardant on the inflorescence of Tagetes patula four weeks after spraying with growth retardant (49 days after transplanting)

\begin{tabular}{|c|c|c|c|c|c|}
\hline Cultivar & Substrate & Chlormequat & $\begin{array}{c}\text { Number of } \\
\text { buds }\end{array}$ & $\begin{array}{c}\text { Number of } \\
\text { flower heads }\end{array}$ & $\begin{array}{c}\text { Flower head } \\
\text { diameter }(\mathrm{cm})\end{array}$ \\
\hline \multirow{2}{*}{ Bonanza Flame } & \multirow{4}{*}{ Compost } & CTR*** & $1.05 \mathrm{~d}^{*}$ & $4.1 \mathrm{a}-\mathrm{c}$ & $4.11 \mathrm{e}-\mathrm{j}$ \\
\hline & & $\mathrm{R}^{* *}$ & $1.33 \mathrm{~b}-\mathrm{d}$ & $3.1 \mathrm{~b}-\mathrm{e}$ & $3.94 \mathrm{~g}-\mathrm{k}$ \\
\hline \multirow{2}{*}{ Durango Red } & & CTR & $1.47 \mathrm{a}-\mathrm{d}$ & $3.8 \mathrm{a}-\mathrm{d}$ & $4.07 \mathrm{e}-\mathrm{k}$ \\
\hline & & $\mathrm{R}$ & $2.58 \mathrm{a}-\mathrm{c}$ & $2.5 \mathrm{~d}-\mathrm{f}$ & $3.88 \mathrm{~h}-\mathrm{k}$ \\
\hline \multirow{2}{*}{ Bonanza Flame } & \multirow{4}{*}{ Peat-coconut } & CTR & $1.47 \mathrm{a}-\mathrm{d}$ & $5.0 \mathrm{a}$ & $4.52 \mathrm{c}-\mathrm{g}$ \\
\hline & & $\mathrm{R}$ & $1.83 \mathrm{a}-\mathrm{d}$ & $3.8 \mathrm{a}-\mathrm{d}$ & $4.44 \mathrm{c}-\mathrm{h}$ \\
\hline \multirow{2}{*}{ Durango Red } & & CTR & $2.91 \mathrm{a}$ & $3.3 \mathrm{~b}-\mathrm{e}$ & $4.55 \mathrm{~b}-\mathrm{f}$ \\
\hline & & $\mathrm{R}$ & $2.72 \mathrm{ab}$ & $4.4 \mathrm{ab}$ & $4.52 \mathrm{c}-\mathrm{f}$ \\
\hline \multirow{2}{*}{ Bonanza Flame } & \multirow{4}{*}{ Peat TS1 } & CTR & $2.11 \mathrm{a}-\mathrm{d}$ & $2.1 \mathrm{ef}$ & $5.49 \mathrm{a}$ \\
\hline & & $\mathrm{R}$ & $2.61 \mathrm{a}-\mathrm{c}$ & $2.6 \mathrm{de}$ & $5.12 \mathrm{ab}$ \\
\hline \multirow{2}{*}{ Durango Red } & & CTR & $1.19 \mathrm{~cd}$ & $1.2 \mathrm{f}$ & $4.95 \mathrm{a}-\mathrm{c}$ \\
\hline & & $\mathrm{R}$ & $2.05 \mathrm{a}-\mathrm{d}$ & $2.1 \mathrm{ef}$ & $4.80 \mathrm{~b}-\mathrm{d}$ \\
\hline \multirow{2}{*}{ Bonanza Flame } & \multirow{4}{*}{ Flower soil } & CTR & $1.63 \mathrm{a}-\mathrm{d}$ & $4.4 \mathrm{ab}$ & $4.64 \mathrm{~b}-\mathrm{e}$ \\
\hline & & $\mathrm{R}$ & $2.33 \mathrm{a}-\mathrm{d}$ & $3.6 \mathrm{~b}-\mathrm{d}$ & $4.13 \mathrm{e}-\mathrm{j}$ \\
\hline \multirow{2}{*}{ Durango Red } & & CTR & $1.56 \mathrm{a}-\mathrm{d}$ & $4.4 \mathrm{ab}$ & $4.35 \mathrm{~d}-\mathrm{i}$ \\
\hline & & $\mathrm{R}$ & $1.78 \mathrm{a}-\mathrm{d}$ & $3.9 \mathrm{a}-\mathrm{d}$ & $4.01 \mathrm{f}-\mathrm{k}$ \\
\hline \multirow{2}{*}{ Bonanza Flame } & \multirow{4}{*}{ Lowmoor peat } & CTR & $2.03 \mathrm{a}-\mathrm{d}$ & $2.9 \mathrm{c}-\mathrm{e}$ & $3.84 \mathrm{i}-\mathrm{k}$ \\
\hline & & $\mathrm{R}$ & $1.70 \mathrm{a}-\mathrm{d}$ & $2.7 \mathrm{de}$ & $3.70 \mathrm{jk}$ \\
\hline \multirow{2}{*}{ Durango Red } & & CTR & $1.28 \mathrm{~b}-\mathrm{d}$ & $2.2 \mathrm{ef}$ & $3.50 \mathrm{k}$ \\
\hline & & $\mathrm{R}$ & $1.64 \mathrm{a}-\mathrm{d}$ & $2.6 \mathrm{de}$ & $3.62 \mathrm{jk}$ \\
\hline
\end{tabular}

$*, * *, * * *$ Explanations: see Tabs 3-5 
the mean values were not significantly higher). The peat TS1 also had a positive impact on the number of flower buds, flower heads and their diameter. Only for the Marvel Mixture cultivar did more buds develop on the plants grown in the peatcoconut substrate. Marigolds growing in lowmoor peat were characterized by a smaller diameter of flower heads (Tab. 6).

Four weeks after spraying the Mexican marigold with the growth retardant, significantly shorter plants were observed for the Marvel Mixture cultivar grown in peat TS1 and for the 'Taishan Orange' grown in lowmoor peat $-24.1 \mathrm{~cm}$ and 22.0 $\mathrm{cm}$, respectively, compared with the control plants (Tab. 5). In other cases, no significant effects of chlormequat on the tested plants were observed. Chlormequat accelerated the development of flower heads for 'Taishan Orange' grown in lowmoor peat (from 1.0 to 1.8). Aside from this effect, the use of this growth retardant did not affect the other examined characteristics of either Mexican marigold cultivar (Tab. 6).

After seven weeks of the experiment, it was noted that the different substrates did not affect the height of French marigolds (Tab. 7). The peat TS1 substrate contributed to the development of longer leaves $(55.8-58.6 \mathrm{~mm})$ in both cultivars tested, in comparison with the other substrates. Lowmoor peat decreased leaf length as well as the diameter and number of shoots. There were no plant variety effects on the tested characteristics of the plants, except leaf length in peat-coconut after chlormequat treatment and stem diameter in lowmoor peat (Tab. 7). For both the Bonanza Flame and Durango Red cultivars, growing in peat TS1 decreased the number of flower heads while increasing their diameter. Moreover, 'Durango Red' grown in the peat TS1 substrate formed significantly fewer flower buds (1.19) compared with the peat-coconut substrate (2.91). For 'Bonanza Flame', more flower heads were observed in the peat-coconut substrate (5.0), while for 'Durango Red' this occurred in the flower soil (4.4). Plants grown in lowmoor peat had flower heads with a smaller diameter (Tab. 8).

The grow th retardant significantly reduced (from 22.3 to $16.1 \mathrm{~cm}$ ) the height of 'Bonanza Flame' plants cultivated in the peat-coconut substrate. In the same substrate, chlormequat application increased the leaf length (from 52.8 to $57.7 \mathrm{~mm}$ ) of 'Durango Red'. Apart from that, its use did not affect the other studied characteristics of marigolds (Tabs 7-8).

\section{DISCUSSION}

The range of substrates available today is diverse and adapted to the species of plants and the various stages of their production. Despite this, horticultural production is often based on readymade peat or peat-coconut substrates enriched with mineral fertilizers. These substrates, unlike standard garden topsoil, are easy to use and do not require a long period of preparation.

In the present experiment, an influence of the peat TS1 and peat-coconut substrates was observed on the growth and development of marigolds, which were characterized by increased height, longer leaves and thicker stems in comparison with plants grown in the other substrates. Plants of the Mexican marigold grown in peat TS1 also developed flowers with a larger diameter of flower heads. Other studies had also reported a positive influence of peat-coconut or coconut substrates on the growth and development of plants such as marigold, verbena, zinnia, celosia and impatiens (Awang and Ismail 1997, Dobrowolska and Startek 2003, Herrera-Moreno et al. 2013, Hongpakdee and Ruamrungsri 2015). In our experiment, the tested marigold cultivars were characterized by much better growth and significantly better flowering in comparison with the other substrates used. According to Hou and Yang (2011) and Vujošević et al. (2013), best quality marigold seedlings are produced when peat is the principal component of the growing media. The favourable impact of highmoor peat (together with mineral soil) has also been reported for the growing and flowering of the Corsican hellebore (Henschke et al. 2014). Witcher (2008) described the highmoor peat-based substrate as a basic substrate component in the production of marigolds, and coconut fibre as its very promising substitute.

According to Nazari et al. (2008), a compost mixture can be used as a soil for the production of ornamental plants. Sewage sludge, being a component of substrates, had improved development of geranium (Andre et al. 2002), and compost substrates also allowed proper growth of horned violet cultivars (Dobrowolska and Janicka 2014). In our study, the Mexican marigold cultivars grown in the compost soil formed the highest number of buds, as measured 21 days after transplanting, while no buds were observed at that time on the plants grown in the peat-coconut substrate. Towards the end of our experiment, the differences in the number of buds between the compost soil and the other substrates (including 
peat-coconut) were not significant. Wraga and Placek (2011), cultivating the Egyptian Star Cluster in substrates containing different amounts of compost, indicated the usefulness of a substrate with $20 \%$ compost for the development of the vegetative and generative phase of the plant.

The present experiment demonstrated a negative effect of using lowmoor peat substrate on the growth and development of marigolds. Like Dobrowolska and Startek (2003), Awang and Ismail (1997) had also obtained fewer and less well developed plants in lowmoor peat. This confirms the previous results (Haber 1998) suggesting the low suitability of lowmoor peat, including its use as a component of horticultural soils, due to its tendency to become clogged and lose its structure, as well as the variable properties of source materials.

The flower soil used in the study favourably influenced the French marigold's stem diameter and leaf length, in a similar way to the peat TS1 and peat-coconut substrates. Bachman and Metzger (2008), in their research with the French marigold, had also obtained a similar size of leaves in plants cultivated both in a commercial potting substrate and a substrate amended with $20 \%$ vermicompost.

In commercial gardening practice, the use of growth retardants is one of the basic procedures with the aim of reducing the length of the stems. Plants grown under the influence of a growth retardant also have stimulated root systems and are more compact, with more abundant leaves and more flowers (Latimer 1991, Asgarian et al. 2013).

In the present experiment, no influence of chlormequat on the growth and development of marigolds was observed beyond individual cases. Similar results had been reported in the case of zinnia sprayed with different doses (500-1500 $\mathrm{mg} \mathrm{dm}{ }^{-3}$ ) of chlormequat (Asgarian et al. 2013), which was explained by an early application of the regulator. However, chlormequat applied as a single drench at $1.0 \mathrm{~g} \mathrm{dm}^{-3}$ significantly reduced the height of zinnia plants (Pinto et al. 2005). Studies with marigold by Schroeter and Janowska (2003) reported shorter stems after the application of chlormequat (460 and $690 \mathrm{mg} \mathrm{dm}^{-3}$ ) and flurprimidol (3.79 and $7.58 \mathrm{mg} \mathrm{dm}^{-3}$ ). These retardants also had a positive impact on the tillering of plants. Jerzy and Schroeter (2004) found reduced growth in retardant-sprayed marigolds, with chlormequat $(0.2 \%)$ being more effective than flurprimidol. Latimer (1991) examined the effect of growth retardants on marigold development and reported a low impact of daminozide (5000 ppm) on stem length, whereas spraying with paclobutrazol (10-40 ppm) and ancymidol (200 ppm) resulted in shorter plants (by $24 \%$ ) in comparison with the controls. He obtained similar results for the leaves, which were smaller, but for the application of ancymidol and paclobutrazol.

In the present study, the growth retardant accelerated the development of flower heads in the Taishan Orange cultivar, but only in lowmoor peat. Pushkar and Singh (2012) had also achieved a very rapid emergence and growth of marigold flower buds after spraying with chlormequat (1000 ppm), which they explained by the plants creating a surplus nutrient supply as a result of the reduction in growth and an increase in the number of branches. Branching was also likely to have contributed to a reduction in the diameter of flower heads. A study by Schroeter and Janowska (2003) reported the lack of an effect of chlormequat on the abundance of flowers and the size of flower heads of the French marigold. Their later study concerning daminozide $\left(2550 \mathrm{mg} \mathrm{dm}^{-3}\right)$ reported even an adverse effect on the flowering of French marigold 'Boy Golden' after spraying seedlings with the retardant (Schroeter-Zakrzewska and Janowska 2009). According to Jerzy and Schroeter (2004), chlormequat had no significant effect on the flowering of French marigold, although it did stimulate the formation of more buds. An increased flower yield, without affecting the initiation of flower buds, was also observed in Mexican marigold plants treated with chlormequat (2000 ppm) (Kumar et al. 2011). Differences in the effectiveness of growth retardants among cultivars can depend on their concentrations and environmental and cultural growing conditions.

\section{CONCLUSIONS}

1. Peat TS1 and peat-coconut are the most suitable substrates for the cultivation of the tested marigold cultivars. Plants grown in those substrates are taller, have longer internodes and leaves, and thicker stems than plants grown in other substrates.

2. Lowmoor peat is the least suitable substrate, regardless of the plant cultivar. Marigolds grown in lowmoor peat are characterized by poorer morphological parameters, which result in lower plant quality and decrease their commercial value.

3. The effect of the growth retardant depends on the cultivar and the substrate. Chlormequat 
significantly reduces the height of 'Marvel Mixture' (in peat TS1), 'Taishan Orange' (in lowmoor peat) and 'Bonanza Flame' (in peatcoconut). 'Durango Red' plants (grown in peatcoconut) develop longer leaves after retardant application. The use of chlormequat also accelerates the development of flower heads in 'Taishan Orange' (in lowmoor peat).

\section{FUNDING}

This study was financed by the Polish Ministry of Science and Higher Education, within the project no. 3500/KRO/2013-2015.

\section{AUTHOR CONTRIBUTIONS}

Both authors contributed to this work. M.M. - contributed to the entire process, analysed and interpreted the data, and wrote the paper; R.M. - conducted biometric measurements.

\section{CONFLICT OF INTEREST}

Authors declare no conflict of interest.

\section{REFERENCES}

AbDella E.M., 2014. Effect of cycocel and trinexapacethyl on growth, flowering and chemical composition of Tagetes erecta L. plants. Int. J. Academic Res. 6: 121-126.

Andre F., Guerrero C., Beltaro J., Brito J., 2002. Comparative study of Pelargonium sp. growning sewage sludge and peat mixtures. Acta Hort. 573: 63-69.

Asgarian H., Nabigol A., Taheri M., 2013. Effects of Paclobutrazol and Cycocel for height control of zinnia. Int. J. Agron. Plant Prod. 4: 3824-3827.

Awang Y., Ismail M., 1997. The growth and flowering of some annual ornamentals on coconut dust. Acta Hortic. 45: 31-38.

Bachman G.R., MetzGer J.D., 2008. Growth of bedding plants in commercial potting substrate amended with vermicompost. Biores. Technol. 99(8): 3155-3161.

Dobrowolska A., JanickA A., 2014. Changes in the chemical composition of organic media used in cultivation of garden horned violet (Viola cornuta L.) from the Patiola $F_{1}$ group. J. Elem. 19(4): 959-976.

Dobrowolska A., STARTeK L., 2003. Wpływ niektórych czynników uprawowych na wzrost i kwitnienie odmian niecierpka nowogwinejkiego z grupy Sonic. Zesz. Probl. Post. Nauk Roln. 491: 43-50.

FASCELla G., 2015. Growing substrates alternative to peat for ornamental plants. In: Soilless CultureUse of Substrates for the Production of Quality
Horticultural Crops. Md. Asaduzzaman (Ed.), InTech: 47-67.

HABER Z., 1998. Współczesne metody wykorzystywania torfów w ogrodnictwie ozdobnym. Zesz. Probl. Post. Nauk Roln. 461: 15-29.

Henschke M., Czuchaj P., Szczepaniak S., 2014. Growth and flowering of Helleborus argutifolius (Viviani) grown in pots depending on substrate type. Acta Agrobot. 67(3): 91-96.

Herrera-Moreno A.M, Carranza C.E., ChaconSANCHEZ M.I., 2013. Establishment of propagation methods for growing promising aromatic plant species of the Lippia (Verbenaceae) and Tagetes (Asteraceae) genera in Colombia. Agron. Colomb. 1: 27-37.

HongPakdee P., Ruamrungsri S., 2015. Water use efficiency, nutrient leaching, and growth in potted marigolds affected by coconut coir dust amended in substrate media. Hort. Environ. Biotechnol. 56(1): 27-35.

Hou J., YANG G., 2011. Evaluation of growing medium composition on marigold (Tagetes erecta L.) seedling quality. Acta Hort. 891: 237-240.

Ingelmo F., Cent R., Ibanez M.A., Pomares F., Gracia J., 1998. Use of MSW compost, dried sewage sludge and other wastes as partial substitutes for peat and soil. Biores. Technol. 63: 123-129.

Jerzy M., Schroeter A., 2004. The dynamics of growth and flowering of marigold cultivated from trans-plants treated with different retardants used to leaves and directly to peat-substrate. Acta Sci. Pol. Hort. Cul. 3(1): 11-23.

Kumar A., Kumar J., Mohan B., Singh J.P., RaJbeer RAM N., 2011. Effect of plant growth regulators on growth, flowering and yield of African marigold (Tagetes erecta L.) cv. Pusa Narangi Gainda. Asian J. Hort. 2: 418-422.

Kumar V., Pal M., Singh R.S., Verma R.K., Verma R.B., 2016. Effect of rooting media on propagation of marigold (Tagets erecta L.) through shoot cutting. Environ. Ecol. 34(3): 930-932.

LAtimer J., 1991. Growth retardants affect landscape performance of Zinnia, Impatiens and Marigold. HortScience 26(5): 557-560.

Nazari F., Farahmand H., Eshghi S., Niki M., Eslamzade M., 2008. The effect of different soil amendments on growth and flowering of African marigold (Tagetes erecta L.) 'Queen'. J. Fruit Ornam. Plant Res. 16: 403-415.

Schroeter A., JANOwska B., 2003. Wpływ retardantów stosowanych dolistnie na jakość rozsady aksamitki rozpierzchłej (Tagetes patula L.) i niecierpka waleriana (Impatiens waleriana Hook.). Zesz. Probl. Post. Nauk Roln. 491: 237-244.

Schroeter-Zakrzewska A., JANOWSKA B., 2009. Comparison of various methods of application of growth regulators in the cultivation of Tagetes patula L. 'Boy Golden'. Electr. J. Pol. Agric. Univ. 12(3): 12. 
Pinto A.Ch.R., Rodrigues T.J.D., Leite I.C., BARbosA J.C., 2005. Growth retardants on development and ornamental quality of potted 'Lilliput' Zinnia elegans Jacq. Sci. Agric. (Piracicaba, Braz.) 4: 337345.

PushKar N.C., Singh A.K., 2012. Effect of pinching and growth retardants on flowering and yield of African marigold (Tagetes erecta L.) var. Pusa Narangi Gainda. Int. J. Hort. 2: 1-4.

Vujošević A., Maksimović S., Lakić N., Savić D., 2013. The optimisation of substrata composition for the production of marigold seedlings. Proc. $1^{\text {st }}$ International Congress on Soil Science, XIII
National Congress in Soil Science, Soil-Water-Plant, Belgrade, Serbia, 23-26 ${ }^{\text {th }}$ September: 175-194.

Witcher A., 2008. Whole tree substrate and fertilizer rate in production of greenhouse-grown petunia (Petunia $\times$ hybrida Vilim.) and marigold (Tagetes patula L.) HortScience 43: 700-705.

Wraga K., PlaceK M., 2011. Use of media supplemented with compost containing potato pulp in cultivation of Egyptian star cluster (Pentas lanceolata (Forssk.) Deflers). Ecol. Chem. Eng. A 18(1): 147-154.

Received March 15, 2017; accepted June 12, 2017 